\title{
Acremonium atrogriseum
}

National Cancer Institute

\section{Source}

National Cancer Institute. Acremonium atrogriseum. NCI Thesaurus. Code C119310.

A species of white colored acremonioid fungus in the phylum Ascomycota. A. atrogriseum forms fusoid conidia in long chains. 